\title{
Estado nutricional e ingestão proteica de idosos com doença de Parkinson
}

Nutritional status and protein intake of elderly people with Parkinson's disease

Estado nutricional e ingestión proteica de ancianos con enfermedad de Parkinson

Maritza Dionicia Quino Paredes Guerdão ${ }^{1 *}$, Sonia Maria César de Azevedo e Silva ${ }^{1}$, Maria de Lourdes do Nascimento da Silva ${ }^{1}$, Manuela de Almeida Roediger ${ }^{2}$.

\section{RESUMO}

Objetivo: Determinar o estado nutricional e a ingestão proteica de pacientes idosos com doença de Parkinson (DP) e sua associação com o grau de evolução da DP. Métodos: Estudo transversal desenvolvido com 31 idosos de ambos os gêneros com DP do ambulatório de Neurologia de um hospital público. Foi realizada a avaliação do estado nutricional e utilizado o Recordatório de 24 horas para avaliar o consumo calórico e proteico. Utilizou-se o teste de qui-quadrado de Pearson e o teste não paramétrico de Mann-Whitney para verificar a associação entre as variáveis, pelo software Stata® versão 13.1 Resultados: $61,3 \%$ dos participantes eram do sexo masculino. Quanto ao diagnóstico do índice de Massa Corporal (IMC), observase que $45 \%$ dos pacientes estavam com o peso adequado para a altura e $22,6 \%$ com baixo peso. A média do IMC foi de $26,78 \mathrm{~kg} / \mathrm{m}^{2}$. A ingestão proteica foi superior a $0,8 \mathrm{~g} / \mathrm{kg} / \mathrm{dia}$, recomendação estabelecida pelas DRI (Dietary Reference Intakes) do Institute of Medicine. A média da ingestão proteica foi 1,22 g/ $/ \mathrm{kg} / \mathrm{dia}$, sendo o consumo superior nas três primeiras refeições (desjejum, lanche da manhã e almoço): 50,17 g, cuando comparada à média do consumo nas três últimas refeições (lanche da tarde, jantar e lanche da noite) de 38,37 g, Conclusão: Não foi verificada associação do estado nutricional e ingestão proteica com o estágio da doença e o tempo de evolução da DP.

Palavras-chave: Estado Nutricional, Ingestão Proteica, Doença de Parkinson, Idoso.

\begin{abstract}
Objective: To determine the nutritional status and protein intake of elderly patients with Parkinson's disease (PD) and its association with the degree of PD evolution. Methods: A cross-sectional study was carried out with 31 elderly patients of both genders with PD from the Neurology outpatient clinic of a public hospital. The nutritional status was evaluated, and the 24-hour Recall was used to evaluate caloric and protein intake. The Pearson chi-square test and the non-parametric Mann-Whitney test were used to verify the association between the variables, by Stata ${ }^{\circledR}$ software version 13.1 . Results: $61.3 \%$ of the participants were male. Regarding the diagnosis of the Body Mass Index (BMI), $45 \%$ of the patients had adequate weight for height and $22.6 \%$ had low weight. The mean BMI was $26.78 \mathrm{~kg} / \mathrm{m} 2$. Protein intake was greater than $0.8 \mathrm{~g} / \mathrm{kg} /$ day, as recommended by the Institute of Medicine's Dietary Reference Intakes (DRI). The average protein intake was $1.22 \mathrm{~g} / \mathrm{kg} /$ day, with the highest consumption in the first three meals (breakfast, morning snak and lunch): $50.17 \mathrm{~g}$, when compared to the average consumption in the last three meals (afternoon snack, dinner and evening snack) of $38.37 \mathrm{~g}$. Conclusion: There was no association between nutritional status and protein intake with the stage of the disease and the time course of PD.
\end{abstract}

Key words: Nutritional Status, Protein Intake, Parkinson's Disease, Elderly.

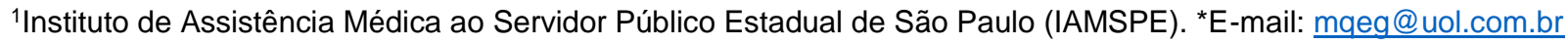

${ }^{2}$ Faculdade de Saúde Pública (FSP), da Universidade de São Paulo (USP).
} 


\section{RESUMEN}

Objetivo: Determinar el estado nutricional y la ingesta proteica de pacientes ancianos con enfermedad de Parkinson (DP) y su asociación con el grado de evolución de la DP. Métodos: Estudio transversal desarrollado con 31 ancianos de ambos géneros con DP del ambulatorio de Neurología de un hospital público. Se realizó la evaluación del estado nutricional y se utilizó el Recordatorio de 24 horas para evaluar el consumo calórico y proteico. Se utilizó la prueba de chi-cuadrado de Pearson y la prueba no paramétrica de Mann-Whitney para verificar la asociación entre las variables, por el software Stata ${ }^{\circledR}$ versión 13.1. Resultados: El $61,3 \%$ de los participantes eran del sexo masculino. En cuanto al diagnóstico del índice de Masa Corporal (IMC), se observa que el $45 \%$ de los pacientes estaban con el peso adecuado para la altura y el $22,6 \%$ con bajo peso. La media del IMC fue de $26,78 \mathrm{~kg} / \mathrm{m} 2$. La ingestión proteica fue superior a $0,8 \mathrm{~g} / \mathrm{kg} /$ día, recomendación establecida por las DRI (Dietary Reference Intakes) del Institute of Medicine. El promedio de la ingestión proteica encontrada fue de $1,22 \mathrm{~g} / \mathrm{kg} /$ día, siendo el consumo superior en las tres primeras comidas (desayuno, refrigerio y almuerzo): $50,17 \mathrm{~g}$, cuando comparada a la media del consumo en las tres últimas comidas (merienda, cena y recena) de 38,37 g, Conclusión: No se verificó asociación del estado nutricional e ingestión proteica con la etapa de la enfermedad y el tiempo de evolución de la DP.

Palabras clave: Estado Nutricional, Ingesta Proteica, Enfermedad de Parkinson, Ancianos.

\section{INTRODUÇÃO}

A Doença de Parkinson é o segundo distúrbio neurodegenerativo mais comum e afeta $1 \%$ da população em todo o mundo após os 65 anos de idade, com uma incidência de 10 a 14 casos por 100.000 por ano (ULAMECK-KOZIOL et al.,2013). No Brasil não existem números exatos, mas estimativas da Associação Brasil Parkinson (ABP) mostram que cerca de 200 mil pessoas são portadoras e, ano a ano, vinte novos casos serão diagnosticados para cada 100 mil pessoas (BRASIL. MINISTÉRIO DE PREVIDÊNCIA SOCIAL, 2009).

A DP é uma doença de progressão lenta, caracterizada por quatro componentes básicos: bradicinesia, tremor de repouso, rigidez e instabilidade postural (GUIMARÃES et al., 2013). É um processo de degeneração específica de neurônios, há aparente correlação com aumento de depósito de ferro na substância negra ou a uma redução de neuromelanina local, um quelante de ferro considerado neuroprotetor (MOON et al., 2016).

O tratamento visa aumentar os níveis de dopamina e aliviar os sintomas, e drogas como levodopa, são usadas. Refeições hiperproteicas associadas à ingestão do medicamento favorecem a interação entre a levodopa e aminoácidos, portanto, a terapia nutricional visa reduzir essa interação (FRACASSO et al., 2013). Como achado comum encontra-se a perda de peso contínua que pode começar antes do diagnóstico da doença e acontecer pela falta de apetite, associada a quadros depressivos frequentes, disfunções sensoriais, dificuldades motoras ou pelo aumento do gasto energético decorrente dos tremores, resultando em um estado de má nutrição nesses pacientes (MORAIS et al., 2013).

A avaliação do estado nutricional, tem como objetivo identificar a presença de distúrbios nutricionais e intervir para promover a recuperação ou manutenção do estado nutricional de indivíduos (MARUCCI et al., 2013). Podem ser utilizados métodos derivados, que associam mais de uma medida antropométrica, como $o$ IMC, que é a relação entre a massa corporal em $\mathrm{kg}$ e a estatura em $\mathrm{m} 2$ (WORLD AND HEALTH ORGANIZATION - WHO 2004). A prevalência de desnutrição é variável e depende do método utilizado para seu diagnóstico e do estágio evolutivo da doença (SHEARD et al., 2013). MORAES et al., (2013) no estudo com 34 pacientes com DP observou que 53\% apresentavam risco de desnutrição, de acordo com a MNA.

O consumo alimentar e proteico é determinado através de inquéritos dietéticos, como o retrospectivo Recordatório de 24 horas, que colhe dados do passado imediato ou de longo prazo (JUZWIAK, 2013), permite avaliar a dieta atual e estima ingestão média de energia e nutrientes específicos e descreve alimentos e hábitos alimentares. As necessidades energéticas diárias são estimadas a partir da taxa de metabolismo 
basal (TMB) para o qual utilizam-se as equações de Harris -Benedict -H\&B, que são específicas para sexo e incluem peso e estatura, A recomendação das necessidades diárias de proteínas, estabelecida pelas DRI (Dietary Reference Intakes) do Institute of Medicine (IOM), é de 0,8 $\mathrm{g} / \mathrm{kg} / \mathrm{dia}$ (MARUCCl et al., 2013).

Identificar o estágio da DP, o género prevalente, o grupo etário, o estado civil, o tempo da doença e a escolaridade, é de fundamental importância para poder identificar as necessidades e definir o tipo de abordagem.

Devido a essas considerações, torna-se imprescindível conhecer as características dos indivíduos idosos, sua condição de saúde, em particular a alimentação e a nutrição, a fim de promover a intervenção adequada, para garantir um envelhecimento saudável e com a melhor qualidade de vida possível. Assim, o objetivo deste estudo foi verificar o estado nutricional e a ingestão proteica de pacientes idosos com (DP) e sua correlação com o grau de evolução da doença.

\section{MÉTODOS}

Trata-se de um estudo transversal de caráter exploratório, composto por 31 voluntários de ambos os gêneros, com idade igual ou superior a 60 anos, diagnóstico clínico de DP, que compareceram em datas préagendadas, para o atendimento no Ambulatório do Serviço de Neurologia do Hospital do Servidor Público Estadual, do município de São Paulo - SP. Foi solicitado o auxílio do responsável ou acompanhante presente na entrevista (cônjuge, filho/a ou outro familiar), quando o paciente foi incapaz de responder a alguma das perguntas.

Foram excluídos do estudo pacientes com limitações físicas para realizar as medidas antropométricas, bem como os que apresentavam outras doenças neurológicas.

As variáveis qualitativas: gênero, estado civil, escolaridade e estágio da doença, assim como as variáveis quantitativas: peso, altura e idade, foram coletadas num formulário padronizado. O peso e a altura corporal foram aferidos no momento da triagem na Balança Antropométrica Digital - Welmy, seguindo técnicas padronizadas. Para a estatura utilizou-se o antropômetro da mesma balança, de acordo com os criterios de Frankfurt (MARUCCl et al., 2013).

As variáveis nutricionais foram o Recordatório de 24 horas e o Índice de Massa Corporal (IMC). As medidas caseiras descritas no Recordatório de 24 horas, foram convertidas em gramas e mililitros e analisadas no Software de Nutrição Avanutriß, para estimar a ingestão energética e proteica. Foi realizado uma única vez, devido à dificuldade dos participantes em retornarem ao ambulatório. Para avaliação da adequação de ingestão proteica, foram utilizadas as DRI do IOM (2011). O requerimento energético foi calculado individualmente pela equação de Harris \& Benedict, com fator atividade 1,3.

A classificação para a avaliação do estado nutricional dos idosos pelo IMC foi conforme os valores propostos pela NSI, 1994 para idosos, que considera subnutrido quando IMC $\leq 22 \mathrm{~kg} / \mathrm{m} 2$, peso adequado IMC $>22$ e $<27 \mathrm{~kg} / \mathrm{m} 2$; e excesso de peso e obesidade IMC $\geq 27 \mathrm{~kg} / \mathrm{m} 2$ (THE NUTRITION SCREENING INITIATIVE - NSI. 1994).

Os dados foram apresentados utilizando-se a estatística descritiva: média, mediana, desvio padrão, valores mínimos e máximos, com respectivos intervalos de confiança para as variáveis quantitativas e porcentagens relativas para as variáveis qualitativas.

O teste de qui-quadrado de Pearson foi utilizado para verificar a associação entre estado nutricional, segundo sexo, grupos etários, estágio e evolução da DP. Para verificar a associação entre a média de ingestão proteica e estágio e evolução da doença, utilizou-se o teste não paramétrico de Mann-Whitney, considerando $p<0.05$. As análises estatísticas foram realizadas com o software Stata® versão 13.1 . 
Este trabalho teve início mediante aprovação do Comitê de Ética em Pesquisa do Instituto de Assistência Médica do Servidor Público Estadual-IAMSPE- São Paulo - SP - sob o Parecer № 186.100. Todos os pacientes participantes do estudo ou seus responsáveis assinaram o "Termo de Consentimento Livre e Esclarecido." - TCLE.

\section{RESULTADOS}

Participaram deste estudo 31 indivíduos com DP, sendo $61,3 \%$ do gênero masculino, $77,4 \%$ casados e $54,8 \%$ cursaram o ensino fundamental, (Tabela 1 ).

Tabela 1: Distribuição de frequência das características da população estudada. São Paulo - SP 2015.

\begin{tabular}{lcc}
\hline Caracterização da população estudada & F & $\%$ \\
\hline Gênero & 19 & 61,3 \\
Masculino & 12 & 38,7 \\
Feminino & & \\
\hline Estado Civil & 24 & 77,4 \\
Casado (a) & 3 & 9,7 \\
Divorciado (a) & 1 & 3,2 \\
Solteiro (a) & 3 & 9,7 \\
Viúvo (a) & & \\
\hline Escolaridade & 17 & 54,8 \\
Fundamental & 10 & 32,3 \\
Médio & 4 & 12,9 \\
Superior & $\mathbf{3 1}$ &
\end{tabular}

Fonte: Autores, 2018. Nota: $\mathbf{f}$ = frequência de ocorrência da variável; \% = porcentagem relativa da variável.

Tabela 2: Distribuição do estado nutricional dos idosos estudados, segundo gênero e grupo etário. São Paulo - SP, Brasil, 2015.

\begin{tabular}{|c|c|c|c|c|c|c|c|}
\hline \multirow{3}{*}{$\begin{array}{l}\text { Variáveis } \\
\text { analisadas }\end{array}$} & \multicolumn{7}{|c|}{ Estado nutricional } \\
\hline & \multicolumn{2}{|c|}{ Subnutrição } & \multicolumn{2}{|c|}{$\begin{array}{c}\text { Peso } \\
\text { adequado }\end{array}$} & \multicolumn{2}{|c|}{$\begin{array}{l}\text { Excesso de } \\
\text { peso/Obesidade }\end{array}$} & \multirow[b]{2}{*}{$P$-valo } \\
\hline & $\mathbf{F}$ & $\%$ & $\mathbf{F}$ & $\%$ & $\mathrm{~F}$ & $\%$ & \\
\hline \multicolumn{8}{|l|}{ Gênero } \\
\hline Masculino & 5 & 71,4 & 7 & 50,0 & 7 & 70,0 & \multirow{2}{*}{0,503} \\
\hline Feminino & 2 & 28,6 & 7 & 50,0 & 3 & 30,0 & \\
\hline \multicolumn{8}{|l|}{ Grupo etário } \\
\hline $60-69$ anos & 6 & 42,9 & 4 & 40,0 & 2 & 28,6 & \multirow{3}{*}{0,924} \\
\hline 70-79 anos & 5 & 35,7 & 4 & 40,0 & 4 & 57,1 & \\
\hline 80 anos e mais & 3 & 21,4 & 2 & 20,0 & 1 & 14,3 & \\
\hline
\end{tabular}

Fonte: Autores, 2018. Nota: $\mathbf{f}$ = frequência de ocorrência da variável; \% = porcentagem relativa da variável. ${ }^{*} P$-valor $<0,05$ referente à estatística Qui-quadrado de Pearson. 
Verificou-se maior prevalência de subnutrição $(71,4 \%)$ nos idosos do gênero masculino e analisando pelo grupo etário, a subnutrição foi mais prevalente $(42,9 \%)$ nos pacientes idosos de 60 a 69 anos. 0 grupo de 70 a 79 anos apresenta maior proporção $(57,1 \%)$ de pacientes idosos com excesso de peso/obesidade (Tabela 2).

Quanto ao consumo calórico e proteico, observa-se que foram superiores ao sugerido (VET) nas três categorias de IMC. O consumo proteico nas três primeiras refeições (desjejum, lanche e almoço), foi superior ao consumo nas outras refeições (lanche da tarde, jantar, lanche da noite) (Tabela 3).

Tabela 3: Análise comparativa das características dietéticas dos idosos, segundo as categorias do IMC. São Paulo - SP, Brasil, 2015.

\begin{tabular}{lcccccc}
\hline & \multicolumn{2}{c}{ Subnutrido } & \multicolumn{2}{c}{$\begin{array}{c}\text { Peso } \\
\text { adequado } \\
\text { à altura }\end{array}$} & \multicolumn{2}{c}{$\begin{array}{c}\text { Excesso de } \\
\text { peso/Obesidade }\end{array}$} \\
\hline Consumo VET/Ptn & Média & DP & Média & DP & Média & DP \\
\hline Ptn Sugerida & 46,01 & 5,74 & 50,20 & 8,26 & 66,83 & 7,34 \\
\hline Ptn Real & 77,00 & 15,47 & 65,51 & 20,38 & 102,81 & 33,79 \\
\hline & & & & & & \\
\hline g/Ptn-D-L-A & 47,05 & 6,90 & 44,57 & 13,55 & 60,96 & 22,27 \\
\hline g/Ptn-L-J-L & 29,93 & 12,08 & 20,90 & 13,46 & 42,91 & 20,86 \\
VET Sugerido & 1531,25 & 196,25 & 1518,21 & 311,98 & 1983,74 & 190,02 \\
\hline VET Real & 1356,27 & 203,63 & 1316,95 & 346,83 & 1743,04 & 510,96
\end{tabular}

Fonte: Autores, 2018. Nota: Média = valor médio da variável, DP = desvio padrão, $\mathbf{P t n}=$ proteína, VET = valor energético total, $\mathbf{g}=$ gramas, $\mathbf{D}=$ desjejum, $\mathbf{L}$ = lanche, $\mathbf{A}=$ almoço, $\mathbf{J}=$ jantar

Tabela 4: Associação do estado nutricional dos idosos estudados, segundo o estágio e o tempo de evolução da doença de Parkinson (DP). São Paulo - SP, Brasil, 2015.

\begin{tabular}{|c|c|c|c|c|c|c|c|}
\hline \multirow{3}{*}{$\begin{array}{l}\text { Variáveis } \\
\text { analisadas }\end{array}$} & \multicolumn{7}{|c|}{ Estado nutricional } \\
\hline & \multicolumn{2}{|c|}{ Subnutrição } & \multicolumn{2}{|c|}{$\begin{array}{c}\text { Peso } \\
\text { adequado }\end{array}$} & \multicolumn{2}{|c|}{$\begin{array}{l}\text { Excesso de } \\
\text { peso/Obesidade }\end{array}$} & \multirow[b]{2}{*}{$P$-valor } \\
\hline & $F$ & $\%$ & $\mathbf{F}$ & $\%$ & $\mathbf{F}$ & $\%$ & \\
\hline \multicolumn{8}{|l|}{ Estágio da DP } \\
\hline 1 a 2 & 8 & 57,1 & 6 & 60,0 & 4 & 57,0 & \multirow{2}{*}{0,989} \\
\hline 3 e mais & 6 & 42,9 & 4 & 40,0 & 3 & 43,0 & \\
\hline \multicolumn{8}{|l|}{ Tempo da DP } \\
\hline 1 a 8 anos & 5 & 35,7 & 5 & 50,0 & 3 & 42,9 & \multirow[t]{2}{*}{0,782} \\
\hline 8 anos e mais & 9 & 64,3 & 5 & 50,0 & 4 & 57,1 & \\
\hline
\end{tabular}

Fonte: Autores, 2018. Nota: $\mathbf{f}$ = frequência de ocorrência da variável; \% = porcentagem relativa da variável.

${ }^{*} \boldsymbol{P}$-valor $<0,05$ referente à estatística Qui-quadrado de Pearson 
Considerando-se o estágio da DP, os pacientes nos estágios iniciais ( 1 a 2 ) apresentaram maior prevalência de subnutrição $(57,1 \%)$. Aqueles com tempo de doença de 8 anos ou mais apresentaram maiores valores de prevalência de subnutrição (64,3\%). Não houve associação entre o estado nutricional, o estágio da doença de Parkinson e o tempo de evolução da doença (Tabela 4).

Realizou-se o teste não paramétrico de Mann-Whitney comparando as médias da ingestão proteica com estágio da DP e tempo de evolução da doença e verificou-se que não houve associação (Tabela 5).

Tabela 5: Análise da ingestão proteica dos idosos estudados, segundo o estágio e o tempo de evolução da doença de Parkinson (DP). São Paulo - SP, Brasil, 2015.

Variáveis analisadas

Ingestão alimentar proteica (g/kg/peso ao dia)

\begin{tabular}{lcc}
\hline & Média da ingestão proteica & Valor de $\mathbf{P}$ \\
\hline Estágio da DP & $83,0(0,22)$ & 0,189 \\
Tempo da DP & $79,0(0,20)$ & 0,358 \\
\hline
\end{tabular}

Fonte: Autores, 2018. P-valor< 0,05 referente à Teste não paramétrico do Mann-Whitney

\section{DISCUSSÃO}

A DP é considerada cosmopolita por estar presente em todas as classes sociais e etnias, acomete homens e mulheres, porém é mais frequente em homens, apesar da população idosa feminina ser maior do que a masculina, provavelmente pelo fator do estresse físico e emocional que os homens enfrentam durante a vida, mas existem estudos que relatam os efeitos neuroprotetores do estrogênio ao longo da vida, contudo o papel do estrogênio como neuroprotetor ainda é controverso (SANTOS e MILAGRES, 2015).

No presente estudo, 61,3\% eram do gênero masculino, enquanto MORAIS et al., (2013) identificaram que $58,3 \%$ eram do gênero masculino. Corroborando essas informações, CARMO E FERREIRA (2016) avaliaram 40 indivíduos com DP e $57,5 \%$ eram do sexo masculino.

Os homens, possuem limiar mais baixo para suportar limitações, dor, desamparo e o fato de, normalmente, desenvolverem suas atividades fora do lar e se apresentarem limitados para o desempenho das mesmas, faz com que percebam limitação de forma geral, enquanto que as mulheres, apesar da diminuição no ritmo do desempenho, continuam a realizar as mesmas atividades (NAVARRO-PETERNELLA e MARCON, 2012).

Este estudo verificou-se maior frequência de subnutrição nos pacientes caracterizados como "idosos jovens" (60-69 anos), enquanto CARMO E FERREIRA (2016) identificaram 37,5\% com baixo peso. No estudo de FRACASSO et al., (2013) com 34 idosos com DP, 53\% apresentaram risco de desnutrição, de acordo com O MNA.

Uma hipótese provável seria o fato de a DP ter acometido esses pacientes precocemente, ou a demora desses indivíduos em procurar o serviço especializado e realizar o diagnóstico definitivo, o que poderia ter comprometido o seu estado nutricional. A subnutrição pode estar relacionada à idade do paciente, pois 0 processo de envelhecimento envolve múltiplas alterações, em particular as nutricionais, que se manifestam por diminuição da estatura, do peso e mudanças significativas na composição corporal, demonstradas pela progressiva redução qualitativa e quantitativa da massa muscular esquelética (FRANÇA e PIVI, 2016). O estado nutricional pode ser comprometido em pacientes com PD, levando à perda de peso devido à baixa ingestão dietética, disfagia, anorexia, redução da absorção e aumento de gasto energético decorrente dos movimentos involuntários (CAPECCI et al., 2013). No entanto não é cabível fazer afirmações considerando o tamanho da amostra. 
Observou-se uma maior frequência de pacientes do gênero masculino com excesso de peso e obesidade (70\%), corroborando estas informações BRICEÑO et al., (2012) identificaram 46,9\% com sobrepeso e 19,2\% obesos, dos 170 pacientes idosos com DP avaliados, enquanto MORAIS et al., (2013) encontraram 33\% da amostra com sobrepeso.

O ganho de peso corporal resulta da desregulação do equilíbrio entre a necessidade energética e a entrada de energia. Na DP, a desregulação pode ser devida a alterações de regulação hipotalâmica, gasto energético ou sinalização dopaminérgica. A interrupção do controle homeostático resulta em aumento do apetite e da fome e pode ser acompanhada por comportamento compulsivo de comer. O ganho de peso, apesar da alimentação inalterada, pode levar a um gasto energético reduzido (KISTNER et al., 2014).

Obesidade e envelhecimento são caracterizados por estado inflamatório sistêmico, de baixo grau, que leva à perda de massa corporal magra, redução da função imunológica, declínio cognitivo, aterosclerose e resistência à insulina. A maioria das substâncias inflamatórias como TNF- $\alpha$ e IL-6 tem efeitos catabólicos sobre o musculoesquelético e está envolvida na sarcopenia, perda involuntária da massa muscular esquelética com o envelhecimento, resultando na redução da capacidade física, redução da mobilidade e fragilidade (TCHERNOF e DESPRÉS, 2013).

A média do consumo proteico foi superior ao recomendado (calculado pelo VET). Dados semelhantes foram encontrados por MORAIS et al., (2013) sendo de 71,2 e 61,9 g/dia. CARMO E FERREIRA (2016), determinaram a ingestão média de 64,2 g/dia.

O consumo proteico em g/Kg/dia, foi de $1,22 \mathrm{~g} / \mathrm{Kg} / \mathrm{dia}$, sendo superior à recomendação das necessidades diárias de proteínas, estabelecida pelas DRI do IOM, de 0,8 g/kg/dia (MARUCCl et al., 2013). Corroborando os resultados, DIAS et al., (2011) identificaram o consumo de $1,13 \mathrm{~g} / \mathrm{Kg} / \mathrm{dia}$ nos portadores de DP do Gruparkinson, com idade entre 45 e 79. LESSA et al., (2011) avaliaram pacientes com DP (45 a 79 anos) e constataram um consumo de $1,13 \mathrm{~g} / \mathrm{kg} / \mathrm{dia}$, enquanto SILVA et al., (2015), encontraram ingestão de 1,1 $\mathrm{g} / \mathrm{kg} / \mathrm{dia}$ em pacientes portadores de DP e controle.

A proteína na dieta permite a promoção da saúde, auxilia na recuperação da doença e mantém a funcionalidade. As pessoas idosas precisam compensar as mudanças no metabolismo das proteínas e as condições inflamatórias e catabólicas associadas a doenças crônicas e agudas que ocorrem comumente com o envelhecimento (BAUER, 2013).

A dietoterapia no parkinsonismo deve levar em consideração o diagnóstico nutricional, o consumo alimentar e a terapêutica médica. Quando se usa a levodopa, fazem-se necessárias medidas dietéticas apropriadas, que poderão contribuir para que esse fármaco se torne eficiente, melhorando a sua biodisponibilidade. Uma ingestão alta de proteína, em horários próximos da medicação, reduz a eficiência da levodopa (GUIMARÃES, 2013), pois tal nutriente compete pela absorção desse fármaco.

O aspecto mais importante da abordagem nutricional é a redistribuição ou a redução da quantidade diária de proteína, resultando em maior eficácia do medicamento o que pode promover melhora na mobilidade de muitos pacientes que experimentam flutuações motoras (JIMÉNEZ et al., 2017). O consumo de proteína, acima das necessidades e em horários máximos da medicação, pode estar associado à falta de informação dos pacientes sobre o tipo de alimentos, quantidade e horários, fato mencionado nas entrevistas por alguns deles.

Sobre o consumo proteico nas refeições, a média foi superior nas três primeiras (café da manhã, lanche e almoço) sendo inadequada a distribuição da proteína ao longo do dia. Corroborando estes dados, CARMO E FERREIRA (2016) observaram maior consumo proteico: 74,7\% entre o café da manhã e o lanche da tarde, assim como no estudo de FRACASSO et al., (2013) houve maior ingestão proteica entre o café da manhã e o lanche da tarde: $70,4 \%$.

Considerando que uma restrição proteica da dieta pode ter efeitos negativos sobre o estado nutricional do paciente, recomenda-se administrar o medicamento uma hora antes da ingestão de alimentos proteicos. Se, 
apesar dessa medida, o paciente apresentar flutuações em seu estado motor em relação à absorção do fármaco, pode-se prescrever uma dieta com consumo proteico diurno restrito e alto teor de proteína no jantar e no lanche (LUIS et al., 2015).

No presente estudo o número de refeições/dia (5 a 6 ) foi superior $(58,1 \%)$ ao encontrado por MORAIS et al., (2013), que revelaram que $36,1 \%$ dos pacientes realizavam 5 refeições/dia e foi inferior ao encontrado por LIMA et al., (2009), no qual constataram (50\%) com 4 refeições/dia.

A reeducação alimentar, o hábito alimentar saudável e a prática de realizar 6 refeições ao dia ajudam a diminuir o apetite, que naturalmente reflete no estado nutricional. No caso da DP, essa fragmentação é necessária para a realização de uma distribuição adequada de nutrientes durante 0 dia, permitindo que 0 medicamento alcance o efeito esperado.

Os idosos costumam fazer a última refeição e se deitar mais cedo e, ao acordar, sentem a necessidade de se alimentar. Consequentemente, o consumo alimentar à tarde e à noite acaba sendo mais reduzido, possivelmente, essa seja a razão de o consumo proteico ser maior nas três primeiras refeições do dia.

Neste estudo, os pacientes apresentaram valores médios do VET real abaixo da média sugerida. Resultados diferentes foram encontrados por MORAIS et al., (2013), que revelou que a ingestão energética ficou acima dos valores médios recomendados; 1632 e $1840 \mathrm{kcal} / \mathrm{dia}$, para homens e mulheres, respectivamente.

A referida ingestão inferior aos valores sugeridos é preocupante, indicando que muitos pacientes, independentemente do estado nutricional, necessitam de monitoramento nutricional e/ou suplementação, para que atinjam suas necessidades diárias e para a manutenção das reservas corporais e do estado nutricional.

A DP é uma patologia que pode sofrer um desequilíbrio na composição corporal devido a que a disfagia e efeitos colaterais das medicações podem limitar a ingestão de alimentos; assim como a própria disfunção motora pode afetar o balanço energético (KISTNER, LHOMMÉE, KRACK, 2014).

O objetivo do suporte nutricional é cobrir as necessidades de energia e nutrientes do paciente com segurança, para prevenir e / ou tratar a desnutrição e suas complicações, adaptando-se à situação do paciente em cada momento de sua evolução e levando em consideração as circunstâncias clínicas e pessoais. Tudo isso para melhorar sua qualidade de vida e prevenir a morbidade e mortalidade (JIMÉNEZ et al., 2017).

Nenhuma diferença significativa foi encontrada entre o estado nutricional com o estágio da doença e tempo de evolução da mesma.

A evolução da DP pode ser determinada por meio da escala Hoehn e Yahr e de outras escalas. São utilizadas de acordo com o perfil da população assistida para definir o tratamento e os protocolos. Podem não ser sensíveis para avaliar o impacto da severidade da doença na vida diária (BEHARI et al., 2005).

Silva et al., (2010), analisaram em 10 indivíduos com DP o comprometimento da qualidade de vida e sua relação com o tempo de evolução e com o estágio de acometimento e observou que o tempo de evolução da doença variou de 2 a 22 anos, sendo que o indivíduo com maior tempo de doença apresentou maior comprometimento, segundo a escala de Hoehn \& Yahr. Porém, o indivíduo com menor tempo de evolução da doença não foi o que apresentou menor índice de gravidade.

No trabalho de Fereshtehnejad et al., (2014) com 143 pacientes com DP e 145 indivíduos do grupo controle iranianos, a pontuação média de Hoehn e Yahr foi de 1,98, apesar de não terem sido realizadas análises de correlação entre as variáveis antropométricas. Nenhuma diferença significativa foi observada, nem em categorias de IMC. 
Van der Marck et al., (2011) realizaram uma meta-análise com base em 12 estudos, para avaliar a relação entre a severidade da doença (HY), a duração e o IMC. Usando regressão, demonstraram que pacientes com DP tiveram um IMC significativamente menor do que os controles, e os pacientes com HY 3 apresentaram menor IMC em comparação com os pacientes com HY2. Esse fato poderia explicar a razão pela qual, na maioria dos doentes estudados em estágio HY 2, não foi encontrada nenhuma diferença entre a média de IMC de pacientes com DP e os do controle. Dados agrupados de sete estudos mostraram que pacientes com Hoehn e Yahr 3 tinham um IMC menor do que os pacientes com HY 2. A duração da doença não foi associada ao IMC.

O presente estudo apresentou algumas limitações que podem ter interferido nos resultados. Em relação à análise do consumo alimentar, os valores encontrados podem estar subestimados ou superestimados, pois as informações foram coletadas uma única vez em decorrência de limitações dos participantes.

Outra limitação deste estudo está associada ao número restrito de pacientes avaliados, provavelmente relacionado ao tipo transversal de delineamento e aos critérios de exclusão estabelecidos. Tais fatores podem ter comprometido a análise estatística de correlação.

Apesar dessas limitações, esse estudo contribuiu para o melhor entendimento e acompanhamento dos pacientes com DP, demostrando a importância da identificação do estágio da doença, assim como a determinação do estado nutricional, consumo alimentar e, em particular, o consumo proteico. No presente trabalho identificou-se um alto consumo proteico nas três primeiras refeições (café da manhã, lanche da manhã e almoço). A redistribuição ou a redução da quantidade diária de proteína resulta em maior eficácia do medicamento, com redução das flutuações motoras e melhora na mobilidade. Tal redistribuição consiste em pequena ingestão proteica no café da manhã, no lanche da manhã e no almoço, e maior ingestão proteica no lanche da tarde e no jantar (JIMÉNEZ, 2017).

Diante das informações obtidas entende-se que é necessário o estabelecimento de uma conduta mais assertiva e individualizada, visando ao atendimento interdisciplinar, o que irá proporcionar melhor qualidade de vida aos pacientes idosos portadores da DP.

Mediante os dados e resultados encontrados, sugere-se a realização de mais estudos que contem com a participação de maior número de pacientes portadores da DP, fazendo uma comparação entre os resultados obtidos e os do grupo controle constituído por indivíduos não portadores da doença.

\section{CONCLUSÃO}

Por meio desta pesquisa foi possível verificar que os pacientes estudados, portadores de DP, apresentaram consumo energético abaixo do recomendado, enquanto o consumo proteico foi superior para todos os grupos (subnutrido, peso adequado para altura e excesso de peso/obesidade), diferindo das recomendações para pacientes acometidos pela DP. Não houve correlação entre o estado nutricional e ingestão proteica com estágio da doença de Parkinson e o tempo de evolução da doença.

\section{REFERÊNCIAS}

1. BAUER J, BIOLO L, CEDERHOLM T, et al. Recomendações baseadas em evidências para a ingestão ideal de proteína dietética em pessoas idosas: um documento de posicionamento do Grupo de Estudos PROT-AGE. J Am Med Dir Assoc. 2013 ago; 14 (8): 542-59.

2. BEHARI M, SRIVASTAVA AK, PANDEY RM. Quality of life in patients with Parkinson's disease. Parkinsonism Relat Disorder. 2005:11\{4\}:221-6.

3. Brasil. Ministério da Previdência Social. Diretrizes de apoio à decisão médico-pericial em clínica médica [Internet]. Brasília, DF; 2009 [citado 2015 Abr 14]. Disponível em: http://www.previdencia.gov.br/arquivos/office/4 091021153135-494.pdf.

4. BRICEÑO HM, ARRIAGA AC, VIOLANTE MR, et al. Overweight is more prevalent in patients with Parkinson's disease. Arq. Neuro-Psiquiatr. vol.70 no.11. São Paulo. Nov. 2012. 
5. CAPECCI M, PETRELLI M, EMANUELLI B, et al. Rest energy expenditure in Parkinson's disease: role of disease progression and dopaminergic therapy. Parkinsonism Relat Disord. 2013;19(2):238-41.

6. CARMO TPS, FERREIRA CCD. Avaliação nutricional e o uso da levodopa com refeições proteicas em pacientes com doença de Parkinson do município de Macaé, Rio de Janeiro. Rev. bras. geriatr. gerontol. vol.19 no.2 Rio de Janeiro Mar./Apr. 2016.

7. DIAS G.G.L, SANTOS KP, VARGAS NML. Avaliação da ingestão de fibras e proteínas por indivíduos portadores da Doença de Parkinson. Nutrir Gerais 2011;5(9):783-97.

8. FRACASSO BM, MORAIS MB, GOMEZ R, et al. Protein intake and the use of levodopa in patients with Parkinson's disease. Rev Chil Nutr 2013; 40(2):102-6.

9. FRANÇA AP, PIVI KGA. Alterações orgânicas, fisiológicas e metabólicas do processo de envelhecimento e seus reflexos na nutrição do idoso. In: Silva do NML, Marucci NMF, Roediger AM. Tratado de Nutrição em Gerontologia. Manole. 2016. p. 49-61.

10. FERESHTEHNEJAD SM, GHAZI L, SADEGHI M, et al. Prevalence of Malnutrition in Patients with Parkinson's Disease: A Comparative Study with Healthy Controlsusing Mini Nutritional Assessment (MNA) Questionnaire. Journal of Parkinson's Disease 4 (2014) 473-481 DOI 10.3233/JPD-130323 IOS Press.

11. GUIMARÃES MPA, SEVERINO VC, PINHEIRO HA. Correlação entre funcionalidade e gravidade da Doença de Parkinson em idosos. Rev Geriatr Gerontol 2013; 7(3): 203-7.

12. INSTITUTE OF MEDICINE; Food and Nutrition Board. Dri Dietary reference intakes: applications in dietary assessment. Washington: National Academy Press; 2001.

13. JIMÉNEZ CT, SÁNCHEZ VS, MEDINA MD, et al. Nutrición en la enfermedad de Parkinson. Nutr Clin Med Vol. XI Número 2 - 2017 pp. 96-113.

14. JUZWIAK CR. Avaliação Dietética. In: Silva SM. Mura JD. Tratado de alimentação, nutrição \& dietoterapia. $2^{\mathfrak{a}}$ ed. São Paulo: Roca: 2013. P163-171.

15. KISTNER A, LHOMMÉE E, KRACK P. Mecanismos de Flutuações do Peso Corporal na Doença de Parkinson. Neurol Frente. 2014; 5: 84. Publicado on-line 2,014 junho 2. prepublished linha 2014 abril 10. doi: [ 10,3389/ fneur.2014.00084 ]

16. LESSA NM, DIAS GG, SANTOS KP. Avaliação do consumo alimentar de fibras e proteínas de indivíduos portadores de doença de Parkinson [abstract]. Nutrire. 2011; 36(Supl.):270. [Apresentado no 11ํㅡㄹ Congresso Nacional da SBAN; 2011 Jun 20 a 23. Fortaleza (CE), Brasil].

17. LIMA RMF, AMARAL AKFJ, AROUCHA EBL, et al. Adaptações na mastigação, deglutição e fonoarticulação em idosos de instituição de longa permanência. Rev. CEFAC vol.11 supl.3 São Paulo.2009.

18. LUIS DA, IZAOLA O, FUENTE B, et al. Enfermedades neurodegenerativas; aspectos nutricionales. Nutr Hosp. 2015;32(2):951-951.

19. MARUCCI MF, ALVES RP, GOMES MM. Nutrição em gerontologia. In: Silva SM, Mura JD. Tratado de alimentação, nutrição \& dietoterapia. $2^{\text {a }}$ ed. São Paulo: Roca; 2013. P.461-488.

20. MOON WJ, PARK JY, YUN WS, et al. A comparison of substantia nigra T1 hyperintensity in Parkinson's disease dementia, Alzheimer's disease and age-matched controls: volumetric analysis of neuromelanin imaging. Korean $\mathrm{J}$ Radiol. 2016; 17:633-40.

21. MORAIS MB, FRACASSO BM, BUSNELLO FM, et al. Parkinson's disease: dietary intake and nutritional status. Rev Bras Geriatr Gerontol 2013; 16(3):503-11.

22. NAVARRO-PETERNELLA FM, MARCON SS. Qualidade de vida de indivíduos com Parkinson e sua relação com tempo de evolução e gravidade da doença. Rev Latino-Am Enferm, Ribeirão Preto. 2012; 20(2):1-8.

23. SANTOS VL, MILAGRES BS. Perfil epidemiológico da Doença de Parkinson no Brasil. Trabalho de conclusão de curso, apresentado no formato de artigo científico ao UniCEUB como requisito parcial para conclusão do Curso de Bacharelado em Biomedicina. Brasilia. 2015

24. SHEARD JM, ASH S, MELLICK GD, et al. Markers of disease severity are associated with malnutrition in Parkinson's disease. PloSOne 2013; 8(3): e57986).

25. SILVA FS, PABIS JV, PABIS C, et al. Evolução da doença de Parkinson e comprometimento da qualidade de vida. Ver Neurocienc 2010;18(4):463-468.

26. SILVA MZC, FRITZEN NC, DE OLIVEIRA M, et al. Protein intake, nitrogen balance and nutritional status in patients with Parkinson's disease; time for a change? Nutr Hosp; 31(6): 2764-70, 2015. MEDLINE | ID: mdl-26040393).

27. TCHERNOF A, DESPRÉS JP. Pathophysiology of human visceral obesity: an update. Physiol Rev. 2013 Jan; 93 (1):359-404. Doi10.1152/physrev.00033.2011.

28. THE NUTRITION SCREENING INITIATIVE - NSI. Incorporating nutrition screening and interventions into medical practice a monograph for physicians. Washington, D. C. US: American Academy of Family Physicians. The American Dietetic Association. National Council on Aging Inc, 1994.

29. ULAMEK-KOZIOL M, BOGUCKA-KOCKA A, KOCKI J, et al. Good and bad sides of diet in Parkinson's disease. Nutrition. 2013;29(2):474-5

30. WORLD HEALTH ORGANIZATION. Physical status: the use and interpretation of anthropometry: report of a WHO expert Committee. Geneva: WHO; 2004.

31. VAN DER MARCK MA, DICKE HC, UC EY, et al. (2012) Body mass index in Parkinson's disease: A meta-analysis. Parkinsonism Relat Disord, 18, 263-267. 